\title{
On Parameter Function of Field - a Register Variable in Translating
}

\author{
Reconstructing Textual Coherence
}

\author{
Li Yingyuan \\ School of Foreign Languages \\ South China University of Technology \\ Guangzhou, Guangdong, 510640, China
}

\author{
Yang Chunlin \\ Vocational College of Xiamen City \\ Xiamen, Fujian, 361008, China
}

\begin{abstract}
It is of great necessity and importance to probe into the function of one of the register variables-field in translating-reconstructing textual coherence. Based on the explication of concrete examples of $\mathrm{E}-\mathrm{C}$ translation, field, as a parameter function of meaning reconstruction in a target text, is hopefully brought to light. This research is thus expected to help a translator or a translation researcher realize that people frequently ignore the relevance between register variables and translation. If the parameter function of field is ignored in the process of translation, the textual function of TT will be greatly different from that of $S T$, as a matter of course.
\end{abstract}

Keywords - translating; register variables; parameter function of field; textual coherence reconstruction

\section{INTRODUCTORY REMARKS}

Both the source text (ST) and the target text (TT) are products of linguistic activities in a certain context. Therefore, the context is one of the most fundamental requisites when a specific text comes into being. Context is referred to as an immediate environment for linguistic activities, so the nature of context is both linguistic and non-linguistic. "According to Gregory \& Caroll, a contextual element consists of three parts: field of discourse, tenor of discourse and mode of discourse, i.e., field, tenor and mode." (Hu Zhuangling, 2005: 274) Field, tenor and mode, therefore, are seen as the most basic elements of register variable which are categorized into some contextual parameters. So far as language is concerned, context is composed by such elements as field, tenor and mode. Field refers to such situational factors as subject matter and settings. Field, however, is an inseparable part of context and one of the conditions where ST or TT is constructed or reconstructed. As an inseparable part of context, field, which produces potential impact on the formation of TT, has long been beyond notice. The textual reconstruction in translating, as a matter of fact, can never do without the involvement of such a variable as field owing to the fact that context is firstly pre-existent, i.e., co-exists with ST; secondly, field needs to be reconstructed in TT. Thus, one of the purposes of building any ST or rebuilding any TT must be based upon the building or rebuilding of field. This realization demonstrates that the interrelation between field and construction of ST or TT is just like the one between tongue and lip. Translating means transforming contextual meaning, indicating that translating must demonstrate the innate field in ST and rebuild it in TT. This viewpoint is fit for the explication of the relationship of inner variables to translational ontology and nature. Considering this, discussion on the parameter function of field should be conducted with the methods based upon the following pre-translation or post-translation analysis and study.

\section{PRE-TRANSLATION ANALYSIS AND STUDY}

For the development of the analysis and study of translation, an utterance selected from the novel Pride and Prejudice by Jane Austen is adopted as follows:"Mr. Bennet was so odd a mixture of quick parts, sarcastic humor, reserve, and caprice, that the experience of three and twenty years had been insufficient to make his wife understand his character (S1). Her mind was less difficult to develop (S2). She was a woman of mean understanding, little information, and uncertain temper (S3). When she was discontented she fancied herself nervous (S4). The business of her life was to get her daughters married (S5); its solace was visiting and news (S6)" (Austen, J., 1992: 4). In the literatures of translation research, many textual methods find their application to pre-translation analysis, one of which is adopted in the process of constructing a ST on the basis of systematic functional grammar. This method may help gain knowledge about the textual function reflected by such contextual parameters as field, tenor and mode. As can be seen from the above, the selected utterance is mainly concerned with the characters' features and the description and summary of the characters' conduct. It is easy to see that field, as a register variable, conspicuously plays a role in the process of textual construction. The following is a case study relevant to the parameter function of field in the ST.This selected utterance intends to give a description about Mr. Bennet and his wife's characters, hobbies or behaviors, or an evaluation and summary of Mr. Bennet and his wife's conducts, ways of treating others, etc. and thus field - one of the register variables reflected in this utterance becomes crystal.It is imaginable that a 'psychological construct' (Sperber \& Wilson, 1998: 15), i.e., a context, naturally emerges from a reader's inner-most when he finishes reading this piece. As can be perceived from the linguistic form, pronouns are frequently used, words are allocated properly and means of sentential connections are employed appropriately, and thus the context 
of situation of the utterance is delineated holistically, reflecting the experiential function of ST, i.e., something occurring around is adequately embodied. The linguistic form involves dynamic and static situations, matters, properties of things, etc. These indicate the author's insight into the characters and their inner worlds. The reason why close semantic chain and logical construction of the utterance are realized is that the author is deft in employing grammatical devices and capable of applying means of lexical cohesion and logical connectors, which is shown by the following facts. In (S1), the author uses nouns like 'Mr. Bennet', 'his wife', 'his character', etc.; in (S2), (S3), (S4), (S5), (S6), and so forth, such pronouns as 'her', 'she', 'herself', 'its', etc., are used. The above-mentioned nouns and pronouns surely provide supportive materialistic condition for the building of one of contextual parameters-field because "Register is a componential part of meaning, usually inclusive of semantic configuration under special conditions, with words and structures embodying these meanings." (Zhang Delu, 2007:19) These materialistic conditions urge the building of context in return, indicating the situation in which causal relationship between context and language thus comes into exitence. Therefore, a so-called text is usually referred to as a semantic unit in which register variables reciprocally reflect in the result of coherence as a glaring feature.

As has been stated above, the utterance is related to the description of Mr. Bennet and his wife's conducts and ways of treating others. The completion of this task owes to appropriate use of words which plays a key role in interweaving cohesive network. The reason why we say so is that the nouns and pronouns in the utterance always cohere with each other, as is clearly shown in the utterance. Besides, such pronouns as 'she', 'her', 'his', etc., which show reiterative relationship, demonstrate the referential relations between 'anaphora' and 'cataphora'. Examples are many like two 'his' ' in (S1), referring to Mr. Bennet; 'his wife' and 'her' in (S1), referring to Mrs. Bennet and thus forming the repetitive relationship; the reiteration of 'herself' in (S4) is reflected in two 'shes'; and the two 'shes' in (S4) and the two 'hers' in (S5) reiterate the 'woman' in (S3); The pronoun 'its' in (S6) repeats 'business' in (S5). As for the relationship of 'anaphora', it is reflected in 'Mr. Bennet' in (S1) and the two 'his' ' in (S1), etc.; the pronoun 'her' in (S2) is utilized to act as an anaphora echoing the antecedent 'his wife' in (S1); Pronouns referred to 'Mrs. Bennet' have reappeared in several places. As major contents of contextual parameter, her image is strengthened which provides information for the theme, causing the configuration of the sense of the utterance to be holistic. There are altogether six sentences in this utterance, but four logical connectors are used. They are 'so...that...' in (S1), indicating the cause-effect relationship, 'and...' in (S1), 'and...' in (S3) showing amplification or addition and 'when...' in (S4), demonstrating time, etc. Considering the contents, the utterance is relevant to the description of characters' personalities, features of individuality and ways of treating others and shows characteristics of delineation of the textual morphology of narrative writing, indicative of the feature that English text pays heed to hypotactic structures. To reflect pre- or post- lexical structure plays an important role in embodying the logical connection of the utterance, which can be perceived from the repetitive use of pronouns. It is proper utilization of grammatical methods, lexical cohesion and logical connectors that the field of the utterance gradually emerges. When the thematic chain of the utterance is established, its contextual configuration and coherent feature come into being smoothly.

It can be postulated that with the help of grammatical devices, lexical cohesion and logical connectors, we get to know that the process of the utterance centers round the construction of one of the register variables - field. Meanwhile, it is under the function of the contextual parameter that the image of the heroine of the story in the utterance is delineated naturally and conspicuously. In this field the personal features of the couple, Mr. and Mrs. Bennets, are conjured up, impressively echoing harmoniously with the specific atmosphere of field. With the narration of the utterance deepening, the psychological construct is reinforced gradually and the parameter function of field in the utterance thus becomes crystal. As one of the contextual parameters, the function of field is paramount since it provides the reader with associable contextual information such as environment, topic and process of communication. This utterance vividly conjures up the images and personal features of the couple, Mr. and Mrs. Bennets: one is an odd, sarcastic, reserved and capricious Mister; the other is a less-informed Mistress of little understanding who has a bad temper, likes complaining about things and worries all day long about her daughters' marriages. The couple has impressively distinctive features of their own. When the utterance is completed, its contextual configuration emerges. The emergence of the process, i.e., emergence of the process of constructing field appears to be natural and smooth. Meanwhile in the process of constructing the text, the impact of field appears in return. This pre-translation textual analysis supplies a reference for the previous translations from English into Chinese. Supposed that post-translation analysis and research are done, we can inspect that whether the existent features of ST is truly reproduced in TT or not, whether the field, the inherent register variable, is transformed or not and whether coherence - the established textual feature, relevance of a certain kind, is reconstructed or not. The following is concerned with textual analysis and study of post-translation.

\section{POST-TRANSLATION TEXTUAL ANALYSIS AND STUDY}

Similar to textual construction, translation is a textual reconstruction which takes its place as a text, i.e., a textual morphology is finally to emerge before the reader. Textual reconstruction cannot do without the participation of field. It is imaginable that the instant the textual reconstruction is involved, the form and content of register variables have to be adjusted, because there is disparity in rules governing the formation and meaning of the two languages. In translating, the process of textual coherence reconstruction, register variables play the same role, which can be clarified by the facts of post-translation analysis. Based on the E-C translation done by LUO Lianggong (2007) and WANG Keyi (1955), differentiation and analysis are conducted so as to see the parameter efficiency of one of the register variables in the process of textual coherence reconstruction. Here are two 
translated versions by LUO Lianggong and WANG Keyi respectively:

\section{Version I}

贝纳特先生就是这么性情古怪复杂，既机敏诙谐、喜 欢冷嘲热讽，又保守矜持，让人捉摸不定，难怪二十三年 的共同生活都不足以让他的妻子真正了解他的性格。而她 的心思却不难理解，她是一个悟性平庸、孤陃寡闻、喜怒 无常的女人。只要遇事不顺心遂意，就臆想着自己神经衰 弱症发作，她平生的大事就是将女儿一一嫁出去，而东走 西访四处打探就成了她精神上的慰藉（bei na te xian sheng jiu shi zhe me xing qing gu guai fu za, ji ji min hui xie, xi huan leng chao re feng, you bao shou jin chi, rang ren zu mo bu ding, nan guai er shi san nian de gong tong sheng huo dou bu zu yi rang ta de qi zi zhen zheng liao jie ta de xing ge. er ta de xin si que bun an li jie, ta shi yi ge wu xing ping yong, gu lou gua wen, xi nu wu chang de nv ren. Zhi yao yu shi bu shun xin sui yi, jiu yi xiang zhe zi ji shen jing shuai ruo zheng fa zuo, ta ping sheng das hi jiu shi jiang nv er yi yi jia chu qu, er dong zou xi fang si chu da tan jiu cheng le ta jing shen shang de wei ji.LUO Lianggong, 2007: 3) .

\section{Version II}

班纳特先生真是个古怪人，他一方面喜欢插科打诨, 爱挖苦人，同时又不苟言笑，变幻莫测，真使他那位太太 积二十三年之经验，还摸不透他的性格。太太的脑子是很 容易加以分析的。她是个智力贫乏、不学无术、喜怒无常 的女人, 只要碰到不称心的事, 她就自以为神经衰弱。她 生平的大事就是嫁女儿; 她生平的安慰就是访友拜客和打 听新闻 (ban na te xian sheng zhen shi ge gu guai ren, ta yi fang mian xi huan cha ked a hun, ai wa ku ren, tong shi you bu gou yan xiao, bian huan mo ce, zhen shi tan a wei tai tai ji er shi san nian zhi jing yan, hai mo bu tout a de xing ge. tai tai de nao zi shi hen rong yi ji yi fen xi de. Ta shi ge zhi li ping yon fa, bu xue wu shu, xi nu wu chang de nv ren, zhi yao peng dao bu cheng xin ru yi de shi, ta jiu zi yi wei shen jing shuai ruo. ta sheng de das hi jiu shi jia nv er. ta sheng ping de an wei jiu shi bai ke he da ting xin wen. WANG Keyi, 1955: 3-4).

Analysis of the parameter function of field in Version I and Version II is performed:

By means of narrative structure, the TT reproduces the Bennets' ways of doing things or conducts, i.e., the Bennets' characters and conducts are reiterated completelyThe two aforesaid translated utterances have realized the transformation of one of the register variables - field in terms of Chinese method of writing, causing TT and its audience to comprehend the realm of being aware of the Bennets' personalities, characters and ways of treating others. Field is known to be inclusive of the whole event described in the original text as well as the speakers' purposeful acts. To reproduce the parameter function of field is the only option. This requirement is an important part in which TT presents field.

Since it gives prominence to form, the English language "lays stress on overt cohesion, sentential forms, structure integration, and manifests its meaning through forms." (LIAN Shuneng, 1993: 48) Therefore, coherence runs through the original text, which is usually quite redundant but writing gets done at one go and thus presents its feature of being coherent. Here is a description concerning the aforesaid Versions I and II. In comparison with ST, TT of Version I seems to have been weakened in its form, but its construction of field has not been affected. So far as its textual function is concerned, there is much correspondence in function between Version I and its ST. The distinguishing feature of the Chinese language is "laying stress on covert coherence, logical order of substances, function and meaning, and the manipulation of spirit over form" (LIAN Shuneng, 1993: 53), since the Mandarin Chinese is a language of ideograph. It is this kind of distinctive feature that Version I manifests some structural features which differ from those of ST. So far as textual function is concerned, TT sufficiently is, however, indicative of nearly the same function. Besides, Version I is different than ST in the application of grammatical devices, lexical cohesion and logical conjunctions, but its thematic chain is retained, the contextual atmosphere of ST is transformed into TT owing to the fact that ST and TT are of the same function, which satiates the 'expectation' of TT readers. In ST, such pronouns as 'she', 'her', 'herself', etc. and conjunctions like 'so...that...', 'and...' and 'when', etc., are used. As a result, ST amply displays the linguistic features of formalization of the English language, i.e., showing that words and sentences are cohesive, thematic chains are tight and an integrated whole of the thematic and ecological environment comes into being. As for $\mathrm{TT}$, the application of pronouns, as can be seen from TT, is not less used than those in ST, but in terms of some special devices of the Chinese language, TT manifests distinctive features of being paratactic in spite of the fact that conjunctions between sentences are not so overt as those we can see in ST, its thematic chain still harbors in the structure of TT, which assures the coherence of the textual gist. In the process of restructuring text, conjunctions such as'既...... 又 ......'(ji ...you ...), ‘ 难 怪 .....'(nan guai ...), ‘而......'(er ...), ‘只要......就......'(zhi yao ...jiu ...), and ‘而......就......'(er ...jiu ...), etc., are utilized. As a result, the textual mechanism is achieved and the parameter function of field is displayed again. Owing to the fact that the parameter function is reconstructed, coordination between field of TT and that of ST naturally comes into existence. The Bennets' personalities, hobbies and ways of treating others are mirrored. With grammatical devices, lexical cohesive methods and logical conjunctions being rationalized, field of TT finally embodies the whole process of the events and the author's acts described in ST. With the matching of register variables in both ST and TT being established, the experiential and logical functions of ST are effectively transformed into TT. In other words, the intrinsic field and established coherence in ST are again constructed in TT.

In comparison with ST, Version II shows that TT has the same function in linguistic formalization. For instance, pronouns used in ST are reflected adequately. As a result, the characteristics of laying stress on the integration of sentential structure, normalization of textual structure, rigorousness in sentential patterns and rigidity or being less elastic are reflected in TT. In addition, the translator makes compensation for ST through Chinese four-word idioms resulting in greater 
readability of TT. Both ST and TT vividly present the Bennets' personalities, hobbies and ways of treating others by using words properly. As can be seen through comparison, we find it not too difficult to see that ST and TT coordinate well with each other in the construction of field and attain an ideal goal. It can thus be postulated that those who finish reading TT may possibly form a clear picture of the contextual configuration or psychological construct in their minds. Accordingly, we can see that Version II has much similarity in form to that of ST and looks inflexible. Furthermore, Version II presents something not matching to the character's status and situations, e.g., 'little information' in ST is transformed into“不学无术”( bu xue wu shu) (ignorant and incompetent) but generally speaking, a projecting pattern for the preexisting coherence has been maintained in TT. As a result of this, both ST and TT have much similarity in textual function. Thus it can be seen that the translator, on the whole, clarifies the parameter function of field in ST and reaches a realm in the function of field in TT as expected. The aspect taken on in the two above-mentioned target texts is inseparable from the translator's exertion to reproduce the parameter function of field in the process of textual reconstruction.

Although the two aforesaid translated utterances have quite a few advantages, their flaws are also glaring. Take the last sentence of the ST for example. There is room to be desired in the translation of the last sentence. Through the postulation of the lexical cohesive relation, we can see that the phrase 'its solace' coordinates with 'the business of her life', i.e., 'its solace' means the same as 'the solace gained from the business of her life'. What business is she doing? The answer is that she wants intensely to get all her daughters married. With the word 'its' instead of 'her' being used, the logical subject is conspicuously 'she'. All that she can do as her normal business is to visit friends and relatives, and to collect news. And then she obtains solace from these activities. The fact reflected in ST can be seen as the goals of her business to get all her daughters married. This is the real sense of her visiting friends and relatives and collecting news. One of the tendencies of thought reflected by the English language is to lay stress on objects, to forsake facts and exclude the involvement of human beings. To a large degree, this kind of situation mirrors a special way of constructing field in the English language. But the two translators transformed the sentence into 而东走西访四处打探就成了她精神上的慰藉 (er dong zou xi fangsi chu da tan jiu cheng le ta jing shen shang de wei ji) or 她生平的安慰就是访友拜客和打听新闻 (ta sheng ping de an wei jiu shi fang you bai ke he da ting xin wen). The two translators just noticed 'her life' but did not see 'the business'. As we can see that 'its solace' and 'visiting and news' do not form a cause-effect relation, but a relation of apposition instead, i.e., 'visiting and news' denotes the content of 'its solace'. Actually, between 'to get her daughters married' and 'its solace' exists a cause-effect relation. Therefore, 'its solace' here is not referred to as 'her life' or 'a whole life of hers'. The above-mentioned translations indicate something arbitrary, instead. Because the translators failed to take into consideration the logical relevance implied in ST, thus the real connotation of TT is far different from that of ST. Hence, the translators availed themselves of the translated version to express subjective volition of their own, which did not correspond with the whole context, and thus failed to mirror the fixed characteristics of the construction of field in ST. When the contextual configuration of ST partially changes in TT, field in TT is no longer the one existing in ST. Coherence in TT is thus not any longer so harmonious when comparison with the coherence in the ST is made. The reason is that the translators did not carefully take contextual parameter function into account. Mismatch of field of register variables of course leads TT readers to misread or misunderstand partial contents of the original. According to the present contextual relationship, the sentence 'its solace was visiting and news' can be transformed into 为女儿婚事忙 碌带来的慰藉就是见到亲朋好友、打听到各种消息 (wei $n v$ er hun shi mang lu dai lai de wei ji jiu shi jian dao qin peng hao you, da ting dao ge zhong xiao xi). Only the translation of this kind can faithfully convey the logical relevance of ST into TT. In a word, the improvement of coherence and realization of projecting the inherent context and established coherence of ST in TT depend on the appropriateness of field reconstruction. The process of textual construction is one in which the author uses every means possible to construct register variables, among which the construction of field is extremely important since the construction of it is beneficial to the construction of the whole context. The construction of field can pave the way for the construction of textual coherence, and thus the textual function is ascertained. So far as translating - a process of textual coherence reconstruction, is concerned, it is a process of restructuring the pre-existing context and established coherence. It not only needs to refer to the pre-existing context and established coherence, but also needs to be restrained by various kinds of textual factors and rules in TT. Hence, at the same time when the requirement of textual feature-coherence and the textual function between texts are maximally satiated, the parameter function of field is naturally the problem to be solved, as a matter of course.

\section{CONCLUSION}

Analysis and exploration have been done of the parameter function of one of register variables - field in combination with translation practice in which textual construction and reconstruction must be involved. From the perspective of parameter function of language, we find that field playing its role in the process of textual translation is well motivated. Pretranslation and post-translation analysis and study make parameter function of field become explicit. The main purpose of this study is to clarify and reveal one of the register variables - field's parameter function in translating - a textual reconstruction activity in order for translators to notice this kind of phenomenon in the process so that field that is closely relevant to theme should not be ignored, because the construction of proper ecological environment beneficial to the reconstruction of theme is sure to lead TT to realize coherence. Besides this, consideration and understanding from the perspective of the parameter function of field help translators get control over the effects of translation when quality in translating is required and evaluated. 


\section{REFERENCES}

[1] Austen, J. 1992. Pride and Prejudice [M]. Wordsworth Editions Ltd.

[2] Sperber, D. \& Deirdre Wilson. 1986. Relevance: Communication and Cognition [M]. Blackwell Publishers.

[3] Zhuanglin, Hu, Yongsheng, Zhu, et al. 2005. General Introduction to Systemic Functional Linguistics [M]. Beijing: Peking University Press.

[4] Guowen, Huang.2006. A Linguistic Exploration into Translation Research-Linguistic Analysis of the English-Chinese Translation of Ancient Chinese Poems and $\mathrm{Ci}[\mathrm{M}]$.Shanhai: Shanghai Foreign Language Education Press.

[5] Shuneng, Lian.1993. A Study on the Contrast Between English and Chinese Languages [M].Beijing: Higher Education Publishing House.

[6] Lianggong, Luo. 2007. Ao Man and Pian Jian (Pride and Prejudice) [Z]. Wuhan: Hubei Changjiang Publishing Group Ltd.

[7] Keyi, Wang. 1955. Ao Man and Pian Jian (Pride and Prejudice) [Z].Shanghai: Shanghai Translation Press.

[8] Zhili, Sun. 2008. Ao Man and Pian Jian (Pride and Prejudice) [Z]. Nanjing: Yilin Publishing House.

[9] Delu, Zhang, et al. 2007. Cohesion in English [Z].Beijing: Foreign Language 